\title{
Pediatric Hypovolemic Shock
}

\author{
Michael J. Hobson ${ }^{1,2}$ and Ranjit S. Chima ${ }^{*, 1,2}$ \\ ${ }^{I}$ Division of Critical Care Medicine, Cincinnati Children's Hospital Medical Center, Cincinnati, Ohio, USA \\ ${ }^{2}$ Department of Pediatrics, University of Cincinnati College of Medicine, Cincinnati Children's Hospital Medical \\ Center, Cincinnati, Ohio, USA
}

\begin{abstract}
Hypovolemic shock is a common yet underappreciated insult which often accompanies illnesses afflicting children. Indeed, it is by far the most common type of shock in the pediatric age group worldwide. Early recognition and treatment of hypovolemic shock is paramount to reversing cellular hypoxia and ischemia before irreparable end-organ damage ensues.
\end{abstract}

Keywords: Hypovolemic shock, dehydration, hemorrhage.

\section{INTRODUCTION}

Hypovolemic shock is a common yet underappreciated insult which often accompanies illnesses afflicting children. Early recognition and treatment of shock is paramount to reversing cellular hypoxia and ischemia before irreparable end-organ damage ensues. Described over 150 years ago, hypovolemic shock remains the most common etiology of shock affecting children today. Diarrheal illnesses resulting in dehydration account alone for approximately $30 \%$ of infant deaths worldwide alone, and it is estimated that 8,000 children younger than the age of five years die every day from untreated dehydration [1]. In the United States, hypovolemic shock still accounts for $10 \%$ of hospital admissions of children less than five years of age [2]. Another major etiology for hypovolemia, especially in the post-operative and traumatic setting is hemorrhage which leads to hemorrhagic shock. This represents a distinct subset of hypovolemic shock.

\section{PATHOGENESIS OF HYPOVOLEMIC SHOCK RELATED TO DEHYDRATION}

The hallmark cardiovascular derangement underlying hypovolemic shock is a reduction in intravascular volume. The resultant loss of preload returning to the heart reduces stroke volume, thus impairing cardiac output. Dehydration, formally defined as a reduction in the water content of the body, is the most common form of hypovolemic shock [3]. Dehydration can be classified according to the degree of fluid deficit, wherein the change in weight is the primary marker for determining the severity of dehydration (Table 1). Furthermore, dehydration may be isonatremic, hyponatremic, or hypernatremic depending upon the changes in serum osmolarity. While most pediatric patients presenting with dehydration will have normal serum sodium values, an accurate history from the patient's caregiver should assess

*Address correspondence to this author at the Division of Critical Care Medicine, Cincinnati Children's Hospital Medical Center, 3333 Burnet Avenue, Cincinnati, OH 45229-3039, USA; Tel: (513) 636-4259;

Fax: (513) 636-4267; E-mail: Ranjit.chima@cchmc.org for the administration of diluted juices or formula which may put the patient at risk for hyponatremia. Hypernatremia results from an excessive loss of free water relative to sodium; the reverse is true in the case of hyponatremic dehydration. The causes of dehydration and hypovolemic shock in children are numerous (Table 2), but can be broadly defined by either decreased intake of fluid, excessive gastrointestinal losses, excessive urinary losses, or translocation of body fluid from the intravascular compartment. Additionally, fever and tachypnea, which accompany many pediatric illnesses, result in hypovolemia as a result of increased insensible fluid losses. Furthermore, infants have many unique physiologic features which may increase their susceptibility to hypovolemic shock, including a relatively higher body water content, an increased metabolic rate, renal immaturity, and the inability to meet their own fluid intake needs independently [3].

\section{CLINICAL PRESENTATION \& LABORATORY EVALUATION OF HYPOVOLEMIC SHOCK IN THE SETTING OF DEHYDRATION}

The clinical presentation of a child presenting in hypovolemic shock are a reflection of the body's attempt to maintain blood flow to vital organs in the face of falling cardiac output as a consequence of a decrease in stroke volume. Activation of the sympathetic nervous system and subsequent release of endogenous catecholamines result in tachycardia; an increased heart rate alone, however, is nonspecific for hypovolemic shock, as fever, agitation, pain, and anemia may be other factors at play in the course of a child's illness. Peripheral vasoconstriction in an effort to maintain coronary and cerebral perfusion manifests as cool distal extremities, weakened distal pulses, and delayed capillary refill. This same increase in systemic vascular resistance allows a child in hypovolemic shock to maintain a normal blood pressure until fluid losses are quite substantial; thus, hypotension should be viewed as a late and ominous sign. Tachypnea in the absence of pulmonary pathology may be compensation for a developing metabolic acidosis. Decreased renal perfusion with activation of the renin- 
Table 1. Degree of Fluid Deficit \& Clinical Symptoms Associated with Dehydration

\begin{tabular}{|l|c|c|c|}
\hline & Mild Dehydration & Moderate Dehydration & Severe Dehydration \\
\hline \hline $\begin{array}{l}\text { Weight Loss } \begin{array}{c}\text { Older child } \\
\text { Infant }\end{array} \\
\text { Heart rate }\end{array}$ & $\begin{array}{c}3 \%(30 \mathrm{ml} / \mathrm{kg}) \\
5 \%(50 \mathrm{ml} / \mathrm{kg})\end{array}$ & $\begin{array}{c}6 \%(60 \mathrm{ml} / \mathrm{kg}) \\
10 \%(100 \mathrm{ml} / \mathrm{kg})\end{array}$ & $\begin{array}{c}9 \%(90 \mathrm{ml} / \mathrm{kg}) \\
15 \%(150 \mathrm{ml} / \mathrm{kg})\end{array}$ \\
\hline Distal pulses & Normal & Mildly increased & Marked tachycardia \\
\hline Capillary refill & Normal & Slightly diminished & Weak, thready \\
\hline Urine output & Normal & Approx. 2 seconds & $>3$ seconds \\
\hline Fontanelle & Normal & Decreased & Anuria \\
\hline Eyes & Flat & Soft & Sunken \\
\hline Tearing & Normal & Diminished & Absent \\
\hline Mucosa & Normal & Dry & Parched \\
\hline
\end{tabular}

Adapted from Gunn VL, Nechyba C. The Harriet Lane Handbook, $16^{\text {th }}$ edition. 2002.

angiotensin-aldosterone axis combined with anti-diuretic hormone $(\mathrm{ADH})$ release from the posterior pituitary will result in diminished urine output. However, urine output may be falsely reassuring in hypovolemic shock secondary to conditions with renal losses such as diabetic ketoacidosis or adrenal insufficiency. As shock progresses, the patient's mental status may evolve from slight irritability to frank lethargy. Other notable physical exam findings consistent with hypovolemia include decreased tearing, dry mucous membranes, and a sunken fontanelle and eyes (Table 1).

Table 2. Causes of Hypovolemic Shock in Children

- Gastrointestinal losses

○ Vomiting

- Diarrhea

- Urinary losses

$\begin{array}{ll}\circ & \text { Diabetic ketoacidosis } \\ \circ & \text { Diabetes insipidus } \\ \circ & \text { Adrenal insufficiency } \\ \circ & \text { Diuretic usage }\end{array}$

- Decreased intake

○ Stomatitis, pharyngitis

- Anorexia, fluid deprivation

- Translocation of body fluids

$\begin{array}{ll}\circ & \text { Small bowel obstruction } \\ \circ & \text { Peritonitis } \\ \circ & \text { Acute pancreatitis } \\ \circ & \text { Burns } \\ \circ & \text { Ascites } \\ \circ & \text { Nephrotic syndrome }\end{array}$

Laboratory evaluation of children with hypovolemic shock will often show a metabolic acidosis with an elevated anion gap $(>14 \mathrm{mEq} / \mathrm{L})$, owing to a switch toward anaerobic metabolism with subsequent lactic acid production. Additionally, a non-anion gap metabolic acidosis may be present if stool output is substantial enough to produce considerable bicarbonate losses. Hypokalemia may also accompany significant gastrointestinal losses. Other laboratory markers of volume depletion include an increased
BUN to creatinine ratio and an elevated urine specific gravity, though again this may be spuriously low in cases of diabetes mellitus, diabetes insipidus and adrenal insufficiency. Of note, early measurement of the patient's serum glucose is warranted, as nearly one-third of patients presenting with hypovolemic shock will have significant hypoglycemia $(<60 \mathrm{mg} / \mathrm{dl})$ [4].

\section{TREATMENT OF HYPOVOLEMIC SHOCK IN THE SETTING OF DEHYDRATION}

Children presenting with mild to moderate dehydration may be candidates for oral rehydration therapy (ORT). Small volumes of rehydration solution are administered intermittently. Appropriate solution for ORT contains approximately $60 \mathrm{mEq} / \mathrm{L}$ of sodium and $2-2.5 \%$ dextrose; the intestinal sodium-glucose transporter absorbs these contents, and water passively follows [3]. Common examples of such fluids include Pedialyte and Rehydralyte. Initial volumes should be 1-2 ml/kg given roughly every five minutes. Volume and frequency are increased as tolerated, up to a total fluid intake of $50 \mathrm{ml} / \mathrm{kg}$ and $100 \mathrm{ml} / \mathrm{kg}$ for mild and moderate dehydration, respectively. A randomized controlled trial of children with moderate dehydration secondary to acute gastroenteritis showed that children treated with ORT had a significantly shorter stay in the emergency department compared to children treated with IV therapy; notably, there was no difference in the rate of hospital admission [5].

Initial stabilization of the pediatric patient in severe hypovolemic shock should proceed as in the treatment of any other critically ill patient. The child's airway and respiratory effort should be supported as necessary, paying special attention to the development of altered mental status and lethargy which may compromise adequate ventilation and oxygenation. Restoration of circulating blood volume remains the cornerstone of therapy for hypovolemic shock. From 1960 to 1990, the advent of intravenous fluid therapy resulted in an eightfold reduction in mortality from hypovolemic shock [1]. As stated previously, early recognition of hypovolemia before the development of hypotension and timely fluid resuscitation prior to the onset of irreversible organ damage are crucial to patient outcome. 
Ideally, fluid administration occurs via a large bore intravenous catheter placed in an upper extremity peripheral vein; if such vascular access is not attainable in an unstable patient, an intraosseous catheter placed in the medial portion of the proximal tibia provides access to the marrow venous plexus. Percutaneous central venous catheters are alternative options for intravascular access, though they are not ideal for rapid fluid resuscitation given their narrow diameter and extensive length.

Isotonic fluids are given as the initial fluid therapy for hypovolemic shock, regardless of the etiology of illness or serum osmolarity. For the patient in hypotensive shock, rapid fluid boluses in $20 \mathrm{ml} / \mathrm{kg}$ aliquots are provided to restore intravascular volume. Lactated Ringer's solution and $0.9 \%$ sodium chloride serve as options for crystalloid fluid. Numerous normal saline fluid boluses may result in a hyperchloremic non-anion gap metabolic acidosis, which may obscure acidosis secondary to poor tissue perfusion. Lactated Ringer's solution has the theoretical benefit of producing bicarbonate from lactate, provided that liver function is normal. Historically, some clinicians have advocated for the role of colloids in the resuscitation of hypovolemic shock, speculating that colloid is more aptly retained in the intravascular space compared to crystalloid fluid. However, a recent multicenter randomized control trial involving a heterogeneous population of adult ICU patients receiving either normal saline or $4 \%$ albumin for intravascular volume resuscitation found no difference in 28 day mortality, rate of organ failure or length of stay [6]. In the pediatric population, Wills et al randomized children suffering from dengue fever who required fluid resuscitation for moderate shock to receive Lactated Ringer's solution, 6 percent dextran 70, or 6 percent hydroxethyl starch [7]. There was no difference in the need for rescue colloid in any of the study groups, noting that crystalloid is effective in fluid resuscitation even in conditions associated with significant capillary leak. Concurrent with fluid resuscitation, attention should be paid to the treatment of hypoglycemia; if present, dextrose is commonly administered as a single $0.5 \mathrm{~g} / \mathrm{kg}$ bolus using $10 \%$ or $25 \%$ dextrose solutions.

Serial physical examination and frequent reassessment of the patient's clinical status are crucial in determining when fluid resuscitation has been adequate. With restoration of intravascular volume, heart rate normalizes, pulse quality improves, and capillary refill should return to below two seconds. Urine output greater than $1 \mathrm{ml} / \mathrm{kg} /$ hour is a marker of adequate renal perfusion. While central venous pressures are not essential to the management of hypovolemic shock, upper extremity central venous catheters, when present, allow for serial CVP measurements, with values of 8-10 mm $\mathrm{Hg}$ indicating adequate fluid repletion. More recently, interest has evolved regarding the use of bedside ultrasound to assess intravascular volume status. Stawicki et al used bedside ultrasound in adult surgical ICU patients to formulate an IVC collapsibility index based on endinspiratory and expiratory diameters of the vessel; these investigators found that low and high IVC-CIs inversely correlated with central venous pressures and thus intravascular volume status [8]. The development of tachypnea, basilar rales, or hepatomegaly after fluid administration suggests that fluid resuscitation has been excessive or concurrent cardiac dysfunction exists. The child with hypovolemic shock requiring more than $60 \mathrm{ml} / \mathrm{kg}$ of fluid resuscitation should raise concern for ongoing losses or an alternative diagnosis, such as a capillary leak syndrome like septic shock.

Beyond their initial fluid resuscitation, children frequently continue to need intravenous fluid therapy for ongoing losses and maintenance requirements until adequate oral intake can be re-established. Care should be taken to avoid iatrogenic-induced hyponatremia during this time period. Patients presenting with shock have several nonosmotic stimuli for ADH production: pain, stress, hypovolemia, hypoglycemia, and a simultaneous respiratory illness are all etiologies for ADH release in the face of low plasma osmolality [9]. Neville et al investigated plasma $\mathrm{ADH}$ concentration in children presenting with viral gastroenteritis requiring hospital admission for IV fluid therapy. Over one-half of the study population was found to have plasma $\mathrm{ADH}$ levels within or above the range associated with maximal antidiuresis, even four hours after fluid administration [10]. Thus, many clinicians advocate for the use of isotonic fluids and close attention to electrolytes in this instance. Fig. (1) summarizes the management of children with dehydration and hypovolemic shock, while Fig. (2) outlines fluid therapy for hyponatremic and hypernatremic dehydration.

\section{HYPOVOLEMIC SHOCK IN THE SETTING OF HEMORRHAGE}

Hemorrhage as a consequence of traumatic injury, gastrointestinal bleeding, severe coagulopathy and intraoperative blood loss may result in the development of hypovolemic shock. In addition to the decrease in intravascular volume and its impairment on stroke volume, the loss of red blood cells significantly reduces oxygen delivery to the tissues. Additionally, hemorrhagic shock and resuscitation may result in the development of a systemic inflammatory state. This may lead to the development of multiple organ dysfunction syndrome (MODS).

\section{Trauma}

Traumatic injuries are the leading cause of death in children and adolescents. Blunt injuries are more common than penetrating trauma, leaving the potential for occult injury with continued hemorrhage. Sources of traumatic bleeding include severe lacerations with external blood loss, intra-abdominal solid organ and retroperitoneal hemorrhage, long bone and pelvic fractures, and traumatic hemothorax. Notably, isolated head trauma alone does not precipitate shock. The injured child with tachycardia and poor peripheral perfusion is in hemorrhagic shock until bleeding can definitively be excluded. As noted above, hypotension is a late and ominous sign-pediatric patients can lose up to $30 \%$ of their blood volume before a fall in blood pressure is observed. Additionally, the serum hematocrit may initially be normal in the setting of acute blood loss.

Resuscitation of the pediatric patient suffering from traumatic hemorrhage proceeds as outlined above for therapy of hypovolemic shock. Additionally, any evident external hemorrhage is controlled with direct pressure. Fluid 


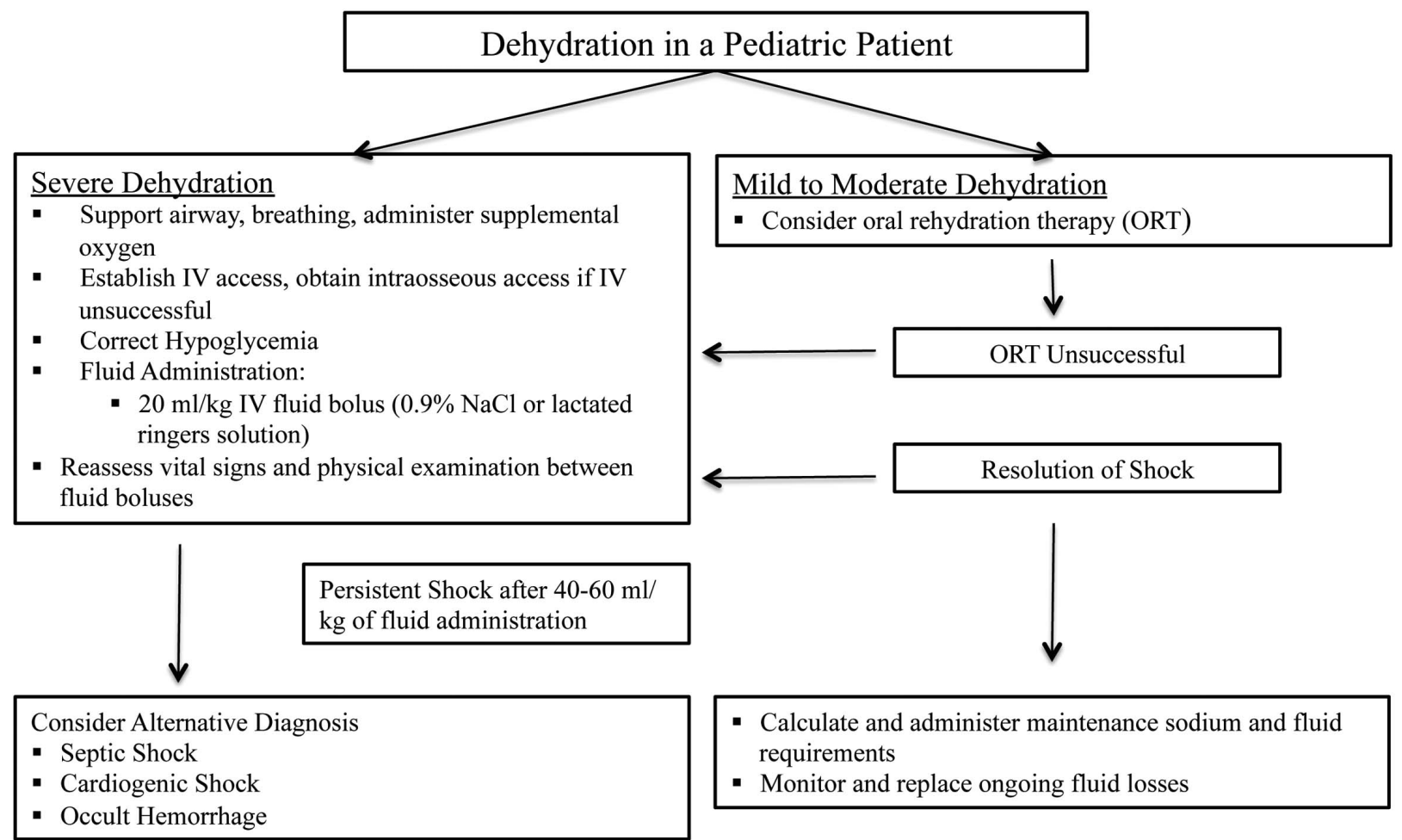

Fig. (1). Treatment algorithm for dehydration in pediatric patients.

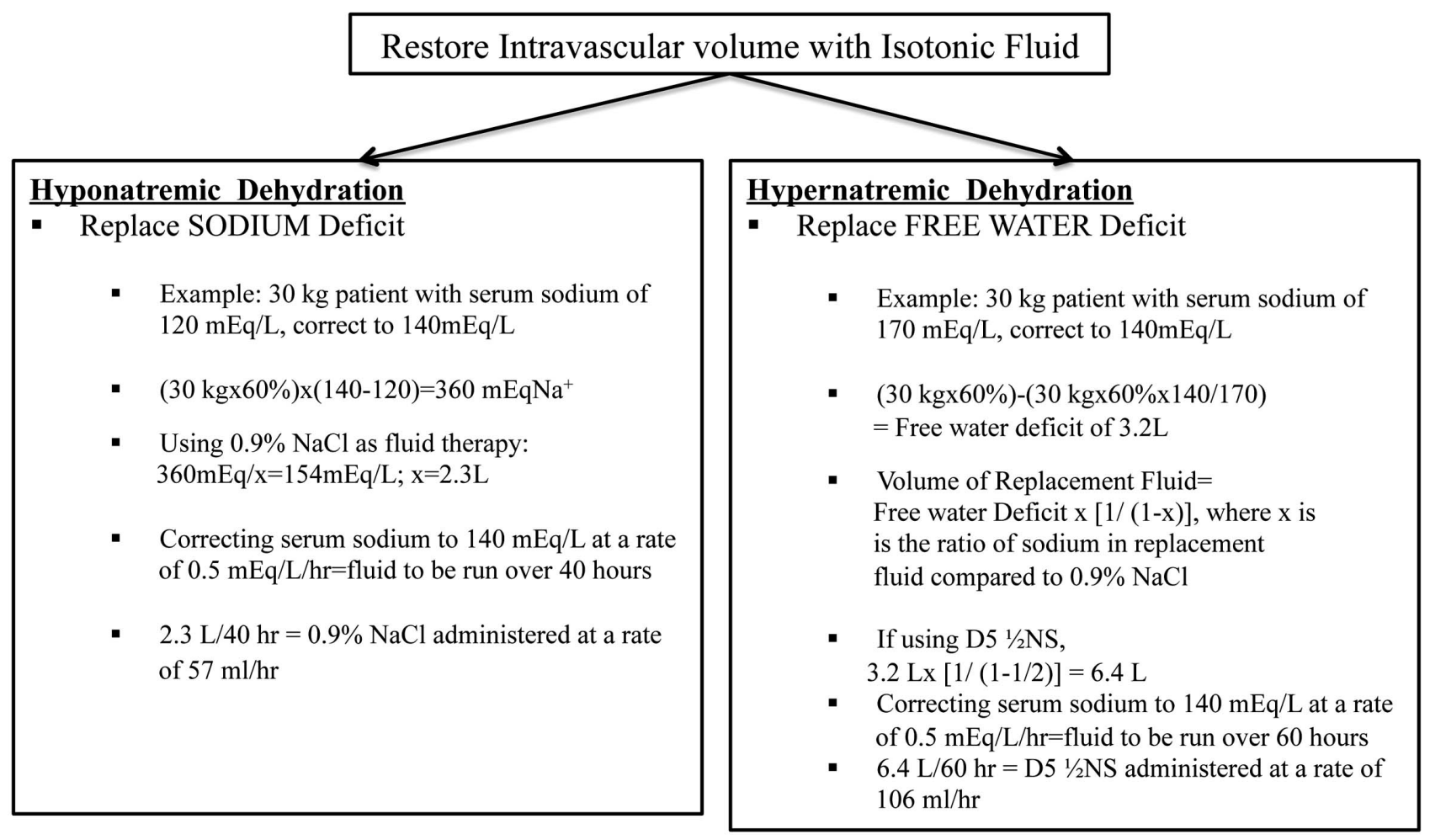

Fig. (2). Management of hyponatremic \& hypernatremic dehydration.

resuscitation again is ideally undertaken with two large bore peripheral upper extremity intravenous catheters. The persistence of shock after two crystalloid boluses often merits transfusion of packed red blood cells. Fully crossmatched, warmed blood is optimal therapy; however, type
O-negative products may be used when clinical instability does not allow time for appropriate cross-matching. Failure to improve after transfusion possibly warrants operative intervention. Other diagnoses, such as cardiac tamponade, tension pneumothorax, and myocardial contusion, should 
also be considered when shock persists after sufficient volume administration.

One of the leading causes of morbidity and mortality after multisystem trauma is the development of multi-organ dysfunction and ARDS secondary to an aberrant inflammatory response. Previous in vitro studies have suggested that hypertonic fluids may favorably alter the activity of inflammatory cells [11]. However, a single center double-blind randomized control trial of adult patients with blunt trauma and pre-hospital hypotension showed no difference in 28-day ARDS-free survival between patients initially resuscitated with Lactated Ringer's solution versus $7.5 \%$ saline with $6 \%$ dextran 70 [12].

Another concept emerging in the field of traumatic hemorrhage is that of permissive hypotension. Traditional fluid resuscitation for traumatic injury has been based on early restoration of blood volume and improvement in mean arterial pressure in order to optimize organ perfusion. The theory of permissive hypotension, or "low-volume fluid resuscitation," argues that aggressive fluid administration raises central venous pressure, disrupts early clot formation, and thus may worsen hemorrhage. A period of suboptimal organ perfusion is tolerated until hemostasis is achieved. To date, no randomized controlled trials have investigated the efficacy of low-volume fluid resuscitation.

\section{Gastrointestinal Bleeding}

Gastrointestinal bleeding is another potential etiology for severe hemorrhagic shock. The sources of gastrointestinal bleeding in children are numerous (Table 3), but can be categorized anatomically according to location relative to the ligament of Treitz. Upper gastrointestinal bleeding, proximal to the ligament, most commonly presents as hematemesis and melena, though if massive can manifest as hematochezia. Care should be taken to exclude bleeding from the patient's nose and respiratory tract in this situation. Lower gastrointestinal bleeding, occurring distal to the ligament of Treitz, can result in either melena or hematochezia depending on the rapidity of hemorrhage. Of note, a significant amount of blood loss may occur within the bowel lumen and remain concealed from direct observation; this possibility should be considered in children with abdominal findings and acute deterioration. Like all forms of hypovolemic shock, acute treatment of gastrointestinal bleeding centers around restoring intravascular volume. Additionally, placement of a nasogastric tube allows for differentiation of upper and lower bleeding as well as the ability to assess for ongoing hemorrhage. Endoscopic evaluation may not only allow a definitive diagnosis of the source of bleeding, but may also provide the opportunity for therapeutic intervention. Gastric acid prophylaxis should be implemented during the patient's treatment to aid in mucosal protection. Other notable pharmacologic options exist specifically for the treatment of variceal bleeding secondary to portal hypertension. Octreotide, a somastatin analogue, is administered as a continuous infusion $(1-2 \mu \mathrm{g} / \mathrm{kg} / \mathrm{hr})$ in efforts to inhibit gastric acid secretion and reduce splanchnic blood flow. Side effects include abdominal cramps, diarrhea, and hyperglycemia. Continuous vasopressin infusions may also reduce splanchnic blood flow for patients suffering from severe gastrointestinal bleeding. Should pharmacologic and non-operative management fail, surgical exploration remains a last option to control hemorrhage.

\section{Table 3. Causes of Gastrointestinal Bleeding in Children}

- $\quad$ Upper gastrointestinal bleeding

- Esophagitis

○ Mallory-Weiss syndrome

- Gastritis

- Gastric/duodenal ulcerations

- Esophageal, gastric varices

○ Arteriovenous malformations

- Lower gastrointestinal bleeding

$\begin{array}{cl}\circ & \text { Milk protein allergy } \\ \circ & \text { Infectious enterocolitis } \\ \circ & \text { Meckel diverticulum } \\ \circ & \text { Intussusception } \\ \circ & \text { Juvenile polyps } \\ \circ & \text { Inflammatory bowel disease } \\ \circ & \text { Henoch-Schonlein purpura } \\ \circ & \text { Hemolytic uremic syndrome } \\ \circ & \text { Arteriovenous malformations }\end{array}$

\section{Massive Transfusion Syndrome}

Regardless of the etiology of hemorrhage, children receiving multiple units of red blood cells are at risk for the massive transfusion syndrome. Massive transfusion is defined in the adult population as receiving ten or more packed RBC units within the first 24 hours of admission [13], whereas in pediatric patients it is typically considered when a child acutely receives more than half of the patient's blood volume (approximately $40 \mathrm{ml} / \mathrm{kg}$ ). Replacement of blood loss with red blood cells alone results in a dilution of platelets and clotting factors, with resultant thrombocytopenia and coagulopathy. To offset these effects, patients being resuscitated with multiple $\mathrm{pRBC}$ transfusions should intermittently receive fresh frozen plasma and platelets as well. Increased ratios of fresh frozen plasma and platelets to packed red blood cells was associated with improved survival in adult trauma patients, though the data may be subject to survivor bias [14]. In addition to dilutional coagulopathy, patients receiving massive transfusion should be monitored closely for hypocalcemia and hyperkalemia.

\section{Recombinant Factor VII}

Recent interest has emerged in the use of recombinant activated factor VII for use in the treatment of refractory hemorrhagic shock. Initially licensed for use in patients with hemophilia having inactivating antibodies to factor VIII and IX, recombinant factor VII acts locally at the site of injury, binding to exposed tissue factor and thereby converting prothrombin to active thrombin. Multiple studies have examined the efficacy of factor VII in controlling acute hemorrhage, often with mixed results. In adult patients undergoing prostatectomy a single preoperative dose of activated factor VII significantly reduced blood loss and transfusion requirements [15]. Similarly, adult patients undergoing non-coronary cardiac surgery had reduced postoperative transfusion requirements when given a $90 \mu \mathrm{g} / \mathrm{kg}$ dose of factor VII upon the discontinuation of 
cardiopulmonary bypass [16]. In the trauma setting, a recent placebo-controlled trial of 143 adults suffering blunt trauma showed that three consecutive doses of rFVIIa significantly reduced transfusion requirements as well as the number of patients requiring massive transfusion; no beneficial effect was shown in penetrating trauma [17]. A larger study of 573 adult trauma patients with ongoing bleeding despite operative intervention were randomized to receive three successive doses of rFVIIa or placebo; patients in the treatment group again had a decreased need for transfusion; no mortality benefit was seen, however [18]. Concern has been raised regarding possible thromboembolic complications in patients receiveing rFVIIa. Particular attention has been paid to adult patients having an underlying propensity to thrombosis. In the field of pediatrics, a recent retrospective review of 135 patients receiving rFVIIa for multiple off-label uses found that three children suffered thrombotic complications, making this a valid concern in the pediatric population as well [19].

\section{CONCLUSION}

In summary, hypovolemic shock is a common malady accompanying a multitude of pediatric illnesses. Early recognition of the signs of shock by the clinician is key to achieving a favorable patient outcome. Again, it should be noted that the appearance of hypotension is a late and ominous sign. Early institution of intravenous fluid resuscitation to restore intravascular volume will ideally reestablish adequate tissue perfusion before end-organ damage ensues. For the patient presenting in hemorrhagic shock, resuscitation often involves repeated blood transfusions, and thus one should be mindful of the pitfalls of massive transfusion. Following successful resuscitation from severe shock, the pediatric patient still may remain at risk for widespread systemic inflammation and subsequent organ dysfunction, and thus diligent care is required in the postresuscitation period.

\section{CONFLICT OF INTEREST}

The authors confirm that this article content has no conflict of interest.

\section{ACKNOWLEDGEMENTS}

Declared none.

\section{REFERENCES}

[1] Thomas NJ, Carcillo JA. Hypovolemic shock in pediatric patients. New Horiz 1998; 6(2): 120-9.
[2] Glass RI, Lew JF, Gangarosa RE, LeBaron CW, Ho MS. Estimates of morbidity and mortality rates for diarrheal diseases in American children. J Pediatr 1991; 118(4 Pt 2): S27-33.

[3] Fleisher GR LS, Henretig FM, Eds. Textbook of pediatric emergency medicine. $5^{\text {th }}$ ed. USA: Lippincott Williams Wilkins 2005.

[4] Tobias JD. Shock in children: the first 60 minutes. Pediatr Ann 1996; 25(6): 330-8.

[5] Atherly-John YC, Cunningham SJ, Crain EF. A randomized trial of oral $v s$ intravenous rehydration in a pediatric emergency department. Arch Pediatr Adolesc Med 2002; 156(12): 1240-3.

[6] Finfer S, Bellomo R, Boyce N, French J, Myburgh J, Norton R. A comparison of albumin and saline for fluid resuscitation in the intensive care unit. N Engl J Med 2004; 350(22): 2247-56.

[7] Wills BA, Nguyen MD, Ha TL, et al. Comparison of three fluid solutions for resuscitation in dengue shock syndrome. N Engl J Med 2005; 353(9): 877-89.

[8] Stawicki SP, Braslow BM, Panebianco NL, et al. Intensivist use of hand-carried ultrasonography to measure IVC collapsibility in estimating intravascular volume status: correlations with CVP. J Am Coll Surg 2009; 209(1): 55-61.

[9] Moritz ML, Ayus JC. Prevention of hospital-acquired hyponatremia: a case for using isotonic saline. Pediatrics 2003; 111(2): 227-30.

[10] Neville KA, Verge CF, O'Meara MW, Walker JL. High antidiuretic hormone levels and hyponatremia in children with gastroenteritis. Pediatrics 2005; 116(6): 1401-7.

[11] Cuschieri J, Gourlay D, Garcia I, Jelacic S, Maier RV. Hypertonic preconditioning inhibits macrophage responsiveness to endotoxin. $\mathrm{J}$ Immunol 2002; 168(3): 1389-96.

[12] Bulger EM, Jurkovich GJ, Nathens AB, et al. Hypertonic resuscitation of hypovolemic shock after blunt trauma: a randomized controlled trial. Arch Surg 2008; 143(2): 139-48; discussion 49.

[13] Spinella PC, Holcomb JB. Resuscitation and transfusion principles for traumatic hemorrhagic shock. Blood Rev 2009; 23(6): 231-40.

[14] Cinat ME, Wallace WC, Nastanski F, et al. Improved survival following massive transfusion in patients who have undergone trauma. Arch Surg 1999; 134(9): 964-8; discussion 8-70.

[15] Friederich PW, Henny CP, Messelink EJ, et al. Effect of recombinant activated factor VII on perioperative blood loss in patients undergoing retropubic prostatectomy: a double-blind placebo-controlled randomised trial. Lancet 2003; 361(9353): 2015.

[16] Diprose P, Herbertson MJ, O'Shaughnessy D, Gill RS. Activated recombinant factor VII after cardiopulmonary bypass reduces allogeneic transfusion in complex non-coronary cardiac surgery: randomized double-blind placebo-controlled pilot study. $\mathrm{Br} \mathrm{J}$ Anaesth 2005; 95(5): 596-602.

[17] Boffard KD, Riou B, Warren B, et al. Recombinant factor VIIa as adjunctive therapy for bleeding control in severely injured trauma patients: two parallel randomized, placebo-controlled, double-blind clinical trials. J Trauma 2005; 59(1): 8-15; discussion -8.

[18] Hauser CJ, Boffard K, Dutton R, et al. Results of the CONTROL trial: efficacy and safety of recombinant activated Factor VII in the management of refractory traumatic hemorrhage. J Trauma 2010; 69(3): 489-500.

[19] Alten JA, Benner K, Green K, Toole B, Tofil NM, Winkler MK. Pediatric off-label use of recombinant factor VIIa. Pediatrics 2009; 123(3): 1066-72. 